\title{
Toll-Like Receptors and Renal Disease
}

\author{
Michael G. Robson \\ Medical Research Council Centre for Transplantation, Kings College London School of Medicine, London, UK
}

\section{Key Words}

Autoimmune diseases · Glomerulonephritis · Inflammation • Ischaemia $\cdot$ Toll-like receptors

\begin{abstract}
Toll-like receptors (TLRs) play a central role in the response of both the innate and the adaptive immune system to microbial ligands. There is also evidence that they are stimulated by endogenous ligands. In this review, I discuss evidence that they are important in renal disease. This discussion considers the role of both endogenous and $\mathrm{mi}-$ crobial ligands, and also the contribution of TLRs present on leucocytes and on intrinsic renal cells. There is strong evidence of a role for TLR2 and TLR4 in renal ischaemia-reperfusion injury, with the effects probably mediated by endogenous ligands. In systemic lupus erythematosus, stimulation of TLR7 and TLR9 by host-derived nucleic acids is important. TLR7 stimulation exacerbates disease, but the role of TLR9 is complex. I also discuss evidence that they are important in other forms of glomerulonephritis, with evidence derived mainly from experimental models in which exogenous ligands have been administered.
\end{abstract}

Copyright $\odot 2009$ S. Karger AG, Basel

\section{Introduction}

Toll-like receptors (TLRs) are named after the Drosophila protein Toll with which they share structural homology. In Drosophila, this protein plays a role in antifungal immunity and pattern formation. TLRs have an extracellular domain comprising leucine-rich repeats and a cytoplasmic domain that has homology with the IL-1 receptor. Only a brief introduction to TLRs is provided here, and the reader is referred to one of many general reviews on TLRs. The first TLR to be defined was TLR4, when a mutation in this gene was shown to be responsible for lipopolysaccharide (LPS) resistance in two separate mouse strains. The family then expanded to the current level of 11 human and 13 mouse members. The TLRs are expressed on a range of leucocytes, including dendritic cells, neutrophils, monocytes, $\mathrm{T}$ cells and $\mathrm{B}$ cells, in addition to non-leucocyte tissue cells. In general, TLR stimulation leads to activation of both the innate and adaptive immune system. The adaptive immune system is influenced by the role of TLRs in mediating dendritic cell maturation and activation, and more recently direct effects on both T and B cells have been described. TLRs recognise a range of pathogen-associated molecular patterns, in addition to the more recent description of several host-derived endogenous ligands. Microbial and endogenous ligands for these TLRs are shown below in

Medical Research Council Centre for Transplantation

Kings College London School of Medicine

Guy's Hospital, London SE1 9RT (UK)

Tel. +44 207188 6768, Fax +44 207188 5660, E-Mail michael.robson@kcl.ac.uk 
Table 1. List of many of the known microbial, synthetic and endogenous ligands for TLRs

\begin{tabular}{|c|c|c|}
\hline & Microbial/synthetic ligands & Endogenous ligands \\
\hline TLR1 ${ }^{\mathrm{a}}$ & triacyl lipopeptides, lipoarabinomannan & \\
\hline TLR2 & peptidoglycan, zymosan & Hsp60, Hsp70, Gp96, HMGB1, urate, hyaluronan \\
\hline TLR3 & dsRNA & mRNA \\
\hline TLR4 & LPS & $\begin{array}{l}\text { Hsp60, Hsp70, Gp96, HMGB1, urate, fibrinogen, } \\
\beta \text {-defensin, surfactant protein A, Tamm-Horsfall protein, } \\
\text { hyaluronan, heparan sulphate }\end{array}$ \\
\hline TLR5 & flagellin & \\
\hline TLR6 $^{\mathrm{a}}$ & diacyl lipopeptides, lipoteichoic acid & \\
\hline TLR7 & ssRNA imidazoquinolines & RNA/protein complexes \\
\hline TLR8 & ssRNA imidazoquinolines & \\
\hline TLR9 & CpG DNA & chromatin \\
\hline TLR10 & unknown & \\
\hline TLR11 & profilin & \\
\hline
\end{tabular}

This list is not exhaustive. ssRNA imidazoquinolines are synthetic compounds. TLR8 is non-functional (or poorly functional) in mice. TLR10 is only present in humans and TLR11 only in mice.

a As dimers with TLR2.

table 1. It should be noted that several investigators have questioned the role of endogenous ligands for TLRs, and argued that the data in many of these reports are the result of contamination with microbial-derived TLR ligands. TLRs may be divided into those that are found on the cell surface, such are TLR4, TLR2 (which forms dimers with TLR1 or TLR6) and TLR5. These receptors recognise bacterial or fungal molecules, in contrast to the intracellular receptors that recognise nucleic acids. This latter group includes TLR3, TLR7, TLR8 and TLR9. A range of intracellular adaptor molecules mediate the effect of TLR stimulation. Myeloid differentiation factor 88 (MyD88) is a key molecule, and is important for all of the TLRs except TLR3. Other signalling molecules are shared by certain TLRs. Toll-IL-1R domain-containing adaptorinducing IFN- $\beta$ (TRIF) mediates the effects of TLR 3 and TLR4. Myd88 adaptor-like (MAL), also known as Toll/ IL-1R domain-containing adaptor protein (TIRAP), mediates effects of TLR2 and TLR4. TRIF-related adapter molecule (TRAM) also mediates effects of TLR4. Figure 1 shows the location and some of the main adaptor molecules involved in TLR signalling.

In addition to expression on leucocytes, TLRs are expressed on parenchymal cells. Renal disease could therefore be influenced by stimulation of TLRs on leucocytes or by stimulation of TLRs on renal cells. Specific examples of the latter will be given below. Primary cultures of mouse renal tubular epithelial cells express TLR1-TLR4 and TLR6 [1]. Cultured mouse mesangial cells show this same pattern of TLR expression [2-4]. These studies on cultured cells are of value, but of more interest is TLR expression in vivo, as demonstrated on intact tissue sections. Immunohistochemical or immunofluorescence studies of TLRs have been difficult however, and this may be due to the low levels of expression, which do not preclude a strong biological effect. A limited number of studies have reported TLR mRNA or protein expression in renal tissue. TLR2 and TLR4 mRNA was expressed on murine renal tubular cells and upregulated by ischaemia [5]. Within glomeruli, TLR4 mRNA was found in podocytes, Bowman's capsule and also in the mesangium [4]. Constitutive expression of TLR2 protein in normal mouse and human kidney has been described on both tubular cells and glomerular endothelium [6]. TLR4 protein has been reported in glomerular podocytes [7] and there has also been a report of TLR3 protein localisation in mouse mesangial cells [8]. In the remainder of this review, I will discuss specific examples of renal disease where the role of TLRs has been assessed.

\section{Ischaemia-Reperfusion Injury}

Ischaemic kidney injury occurs when kidneys temporarily receive an inadequate blood supply and this can occur in both native and transplanted kidneys. Mouse models of ischaemia-reperfusion injury have been developed in which the renal artery is clamped for a specified time. There is strong evidence for a role for TLR2 and TLR4 in these models. TLR2-deficient mice were shown 


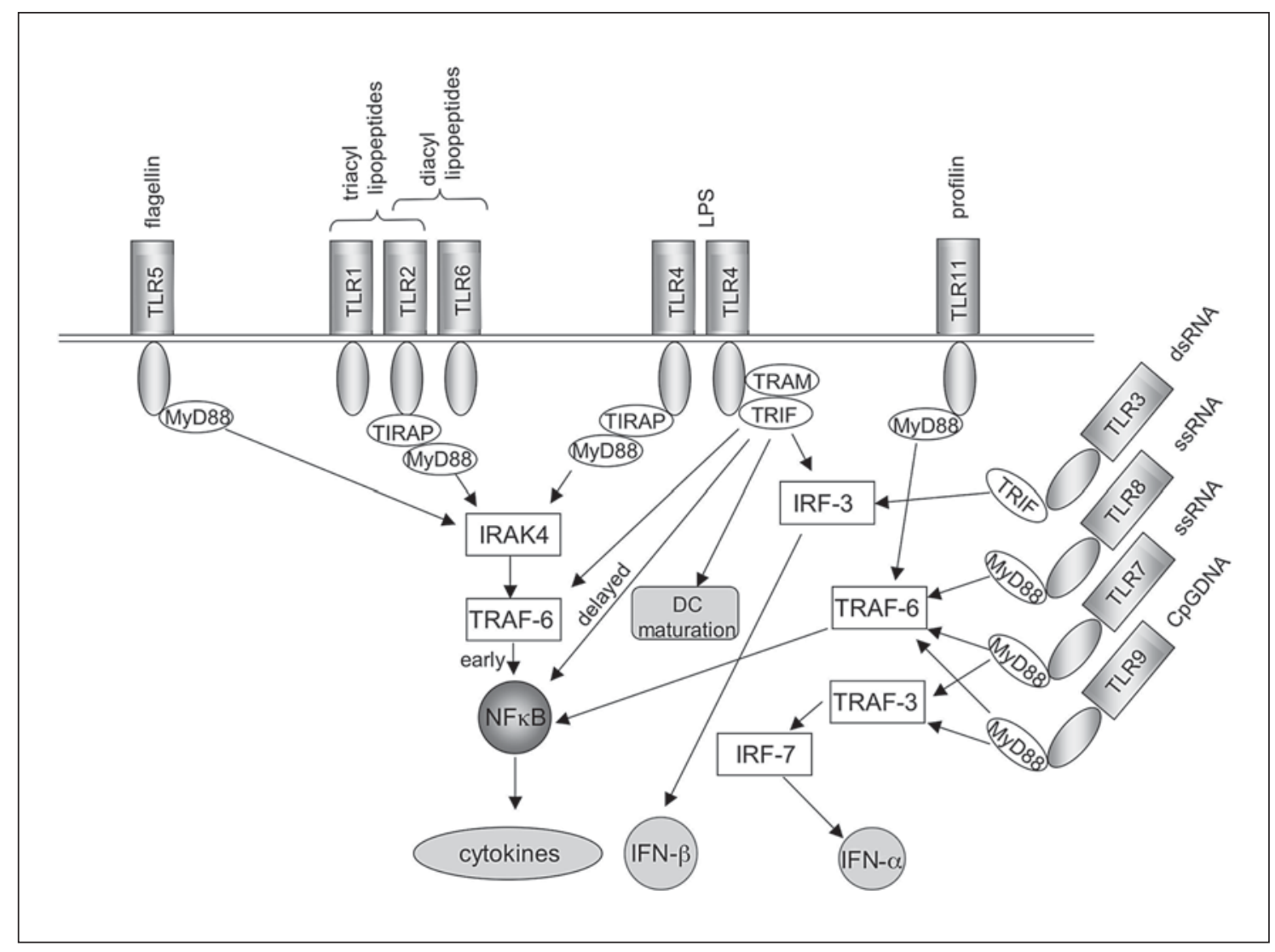

Fig. 1. TLR signalling pathways. TLR1, TLR2, TLR4, TLR5, TLR6, TLR7, TLR8, TLR9 and TLR11 signal via MyD88-dependent mechanisms. In addition, TLR3 and TLR4 signal via MyD88-independent pathways. TLR3 is expressed on the cell surface of fibroblasts, and intracellularly on dendritic cells. Abbreviations used are TRAF-6/-3 = TNF receptor-associated factor 6/3; IRAK = IL-1R-associated kinase; IRF = IFN regulatory factor; $\mathrm{NF \kappa \textrm {B }}=$ nuclear factor $\kappa \mathrm{B} ; \mathrm{DC}=$ dendritic cell.

to be protected from both the functional and histological consequences of ischaemia [9]. In addition, experiments in bone marrow chimeras suggested that the dominant effect was via TLR2 on renal tubular cells. An important role for TLR2 was confirmed in a subsequent study, which also showed that the effect occurred through both MyD88-dependent and -independent pathways [6]. A role for TLRs, again with a dominant role for TLR4, on renal tubular cells was shown, and in this case the effect depended completely on MyD88 [10]. No substances are administered to animals in this model, and this strongly suggests that any effects are due to endogenous ligands. A role for endogenous ligands is further supported by experiments in which sham ischaemia was induced in cultured cells in vitro $[9,10]$. A number of proposed endogenous ligands for TLR2 and TLR4 have been found in ischaemic renal tissue, including high mobility group box
1, hyaluronan, biglycan and heat shock protein 70 , and these could all potentially stimulate TLR2 or TLR4 [10].

\section{Lupus Nephritis}

Disease mechanisms in glomerulonephritis (GN) have been studied using inbred strains of mice that spontaneously develop a disease resembling human systemic lupus erythematosus (SLE). Exogenous ligands that activate TLRs have been shown to exacerbate murine SLE, and this has been used to explain the link between infection and exacerbation of disease in SLE. Lipid A, an agonist for TLR4, was given to a number of SLE-prone strains before TLR4 had been discovered [11]. In all cases, kidney disease was exacerbated, and this was associated with an increase in anti-DNA antibodies and with polyclonal B-cell activation. A series of studies explored the effect of giving known ligands for TLR3, TLR7 and 
TLR9 to MRLlpr mice, in order to mimic the effects of viral infection $[8,12,13]$. All studies showed an increase in kidney disease with TLR stimulation. In the case of TLR3 or TLR7 stimulation, there was no increase in anti-DNA auto-antibodies. This contrasted with the study of TLR9 stimulation, which led to an increase in anti-DNA antibodies. It should be noted that an older study in which bacterial DNA (now known to be a TLR9 agonist) was administered gave a different result. Although auto-antibodies were increased, there was less nephritis. The reason for this difference is not clear, although a different SLE-prone strain and different DNA preparations were used [14]. It should be noted that none of these studies included the use of mice genetically deficient in the relevant TLR. The specific role of a given TLR is therefore assumed but not proven in each of these studies.

A separate consideration from the effect of exogenously administered TLR ligands is that of endogenous ligands. Although TLR9 and TLR7 recognise bacterial DNA and viral RNA, they can also recognise mammalian nucleic acids. Since auto-antibodies to nucleic acidassociated antigens are the hallmark of SLE, the role of TLR7 and TLR9 in the generation of this autoimmunity has been the focus of much research. An elegant series of in vitro studies by Marshak-Rothstein and colleagues revealed a two-receptor paradigm in which auto-antigens containing DNA or RNA bound to antibody on the B cell, and were then delivered to intracellular TLR7 or TLR9 [reviewed in ref. 15]. This leads to a preferential stimulation of B cells reactive with nucleic acid-associated antigens, and may explain the loss of tolerance to nuclear antigens that occurs in SLE. TLR7 and TLR9 within plasmacy toid dendritic cells may also be activated by immune complexes containing RNA or DNA. Immune complexes taken up via dendritic cell Fc receptors may then stimulate intracellular TLR7 or TLR9 if they contain nucleic acid components [15]. This dendritic cell activation may play a role in the pathogenesis of SLE. In this latter model, immune complexes must have formed before these sequences of events can occur, so that tolerance must have already been lost. However, this pathway could provide amplification of disease mechanisms.

The real test of a role for TLR7 or TLR9 in SLE, in the absence of exogenous ligand administration, comes from studies using SLE-prone mice made deficient in TLR7 or TLR9. Disease development is compared with TLR-sufficient controls. Initially there were some conflicting studies on the role of TLR9. However, the most definitive report came in a paper that also described the phenotype of TLR7-deficient SLE-prone mice [16]. The conclusion for TLR9 was that there were less anti-DNA antibodies with no change in anti-Sm/RNP antibodies. Surprisingly there was an increase in mortality in TLR9-deficient mice. This was accompanied by an increase in spleen size, and also an increase in B cell, T cell and plasmacytoid dendritic cell activation. There was an increase in glomerular disease, although the effect was not large. The reasons for this increase in immune activation, mortality and GN, despite the presence of more anti-DNA antibodies, are not clear. The finding of more plasmacytoid dendritic cell activation in TLR9-deficient mice is in conflict with the in vitro data mentioned above [17]. The role of TLR9 in murine SLE in vivo requires further study. For TLR7-deficient SLE-prone mice, there was no change in anti-DNA antibodies with less anti-Sm/RNP antibodies, as might be expected. In addition, there was also less renal disease and less immune activation, although the effect on nephritis was small. There was no difference in glomerular or interstitial disease alone, and only the composite score reached significance. No mortality data were reported. Further insights into the role of TLR7 come from the observation that the Y-linked accelerator locus contains a TLR7 duplication which exacerbates disease in BXSB mice [18]. Subsequent studies have confirmed the importance of the TLR7 duplication in murine SLE, though other genes in the Y-linked accelerator locus may make a contribution.

Two studies have explored the therapeutic potential of TLR7 or TLR9 blockade with synthetic oligonucleotides in murine lupus. In one report, the use of IRS954, a dual TLR7 and TLR9 inhibitor, was protective in the $\mathrm{NZB} \times \mathrm{NZW}$ lupus model and ameliorated both autoantibodies (dsDNA and Smith antigen) and GN [19]. In another report, this same inhibitor decreased GN in MRLlpr mice but had no effect on auto-antibody levels [20]. In contrast, a specific TLR7 antagonist, IRS661, inhibited both auto-antibodies (dsDNA and Smith antigen) and renal disease. These studies highlight the therapeutic potential of TLR inhibitors. However, the observed effects on auto-antibodies do not always correlate with the results expected from the mechanisms suggested by studies in TLR7- and TLR9-deficient mice.

\section{Other GN Models}

Other models used to explore the role of TLRs in GN include nephrotoxic nephritis models, apoferritin-induced nephritis, models of anti-neutrophil cytoplasmic antibody-associated systemic vasculitis (AASV), cryoglobulinaemia and IgA nephropathy. The nephrotoxic 
nephritis model consists of two phases. The heterologous phase is due to the passive glomerular binding antibody, which causes acute inflammation that peaks at $2 \mathrm{~h}$ and resolves by $24 \mathrm{~h}$. The autologous phase is due to an immune response to the foreign antibody and leads to crescent formation, thrombosis and proliferation, developing over weeks. Early reports showed that a small dose of endotoxin could exacerbate the heterologous phase in the rat [17]. Our own studies extended these findings using specific ligands for TLR2 or TLR4, given with glomerular binding antibody $[3,4]$. Bone marrow chimera experiments showed that there was an important role for TLR2 or TLR4 on renal cells in addition to neutrophils. This was clear when assessing neutrophil influx but was less marked for proteinuria, which was more dependent on neutrophil TLRs. These studies did not demonstrate which particular glomerular cell was implicated in these TLR-mediated effects. We also performed a study in which a TLR2 ligand was given at the time of priming with sheep IgG in the autologous nephrotoxic nephritis model [21]. There was an increase in crescentic disease, and an augmentation of the humoral and cellular immune response to sheep IgG. This effect on the adaptive immune response did not clearly follow a Th1 or Th2 pattern, but did suggest that TLR stimulation could convert mild disease into severe disease by modulation of the nephritogenic immune response.

TLRs have also been implicated in the apoferritin model. Apoferritin-induced nephritis is caused by repeated immunisation with this large protein which localises in the mesangium. A TLR9 ligand was given, and shown to skew the immune response towards Th1 and to exacerbate disease [22]. However, the role of TLR9 was assumed as knockout mice were not used. A disease resembling IgA nephropathy develops spontaneously in the ddY inbred mouse. In this strain, TLR9 stimulation with CpG DNA caused Th1 polarisation and exacerbated disease [23]. Furthermore, TLR9 polymorphisms were associated with disease severity in humans with IgA nephropathy. AASV mouse models have recently been developed. These models are not autoimmune, as myeloperoxidase-deficient mice are used for the generation of a pathogenic immune response, with antibody or spleen cells transferred into myeloperoxidase-sufficient recipients. In the AASV model in which passive anti-myeloperoxidase IgG causes disease, LPS was shown to exacerbate pathology, an effect that was partially TNF dependent [24]. Crude LPS which can activate TLR2 in addition to TLR4 was used, and knockout mice were not used to demonstrate a role for a specific TLR.

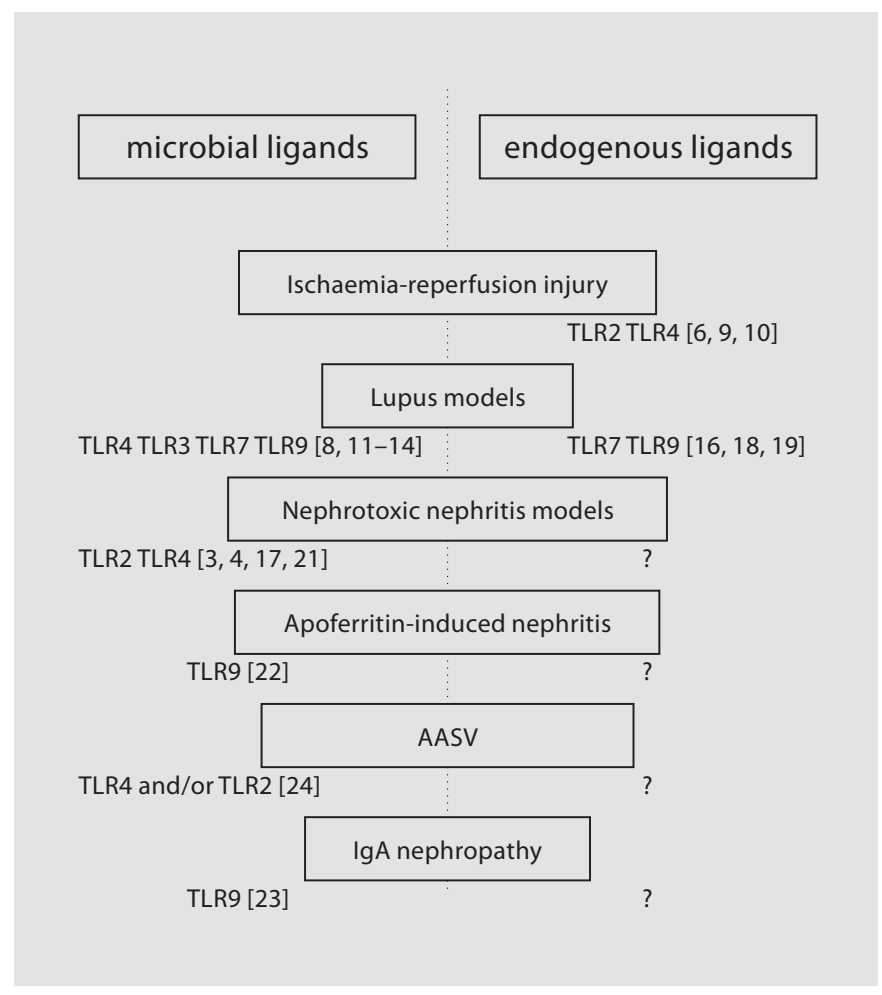

Fig. 2. Diagram showing the models of renal disease where there is evidence of a role for TLRs. The right half of the figure shows TLRs that are probably stimulated by endogenous ligands. The left half shows TLRs that are stimulated by exogenous ligands, mimicking the effect of microbial ligands. Numbers in brackets refer to references in this review. AASV $=$ ANCA-associated systemic vasculitis. Question marks indicate where the role of TLRs is unclear.

To date, there are no data to show that endogenous ligands for TLRs could modulate disease in models of GN other than ligands for TLR7 and TL9 in spontaneous SLE-prone mice, as described above. Many of these models rely on the administration of substances in order to induce disease. As I mentioned earlier and as argued by others, even if substances have undetectable LPS levels, it is difficult to be sure that trace levels of other TLR stimulants are not present. TLR4 has been suggested to be important in a transgenic model of cryoglobulinaemia [7]. This is a spontaneous model of GN which does not require the injection of a substance to initiate disease. In this report, the in vitro podocyte response to fibrinogen (a proposed endogenous ligand for TLR4) was reported and immunostaining showed TLR4 expression in podocytes on histology. However, there were no in vivo data to directly show a role for TLR4 in the development of disease. 


\section{Conclusions}

In this brief review I have summarised data on the role of TLRs in renal disease. Strong evidence comes from ischaemia-reperfusion injury where a role for stimulation of TLR2 and TLR4 on tubular cells by endogenous ligands has been shown. Other available models of interstitial disease include unilateral ureteric obstruction which causes fibrosis. There are no published reports on the role of TLRs in such models. In autoimmune glomerular disease, I have described data suggesting that endogenous ligands for TLR7 and TLR9 are important in the loss of tolerance to auto-antigens in SLE. The in vivo data are consistent for TLR7, but clarification is needed for TLR9. There is ample evidence that exogenous ligands can exacerbate SLE and other types of GN. There are few data on the role of endogenous ligands for TLRs in GN for diseases other than SLE. Figure 2 summarises available data and shows the gaps in our understanding.
An important area of future study will be to establish the extent to which endogenous ligands are important in glomerular diseases in addition to ischaemia-reperfusion injury. Confirmation of which endogenous ligands are relevant will also be needed. Inhibition of TLR2 and TLR4 is a promising therapeutic option for renal ischaemia-reperfusion, particularly in the setting of transplantation. TLR7 and TLR9 are also potential therapeutic targets in SLE, although the increased disease in TLR9deficient SLE-prone mice perhaps makes TLR7 a more likely target than TLR9.

\section{Acknowledgements}

I am grateful to Heather Brown for providing figure 1. Our own work on TLRs has been funded by the Wellcome Trust and Guy's and St. Thomas' Charity.

\section{References}

1 Tsuboi N, Yoshikai Y, Matsuo S, Kikuchi T, Iwami K, Nagai Y, Takeuchi O, Akira S, Matsuguchi T: Roles of toll-like receptors in $\mathrm{C}-\mathrm{C}$ chemokine production by renal tubular epithelial cells. J Immunol 2002;169:20262033.

$\checkmark 2$ Patole PS, Pawar RD, Lech M, Zecher D, Schmidt H, Segerer S, Ellwart A, Henger A, Kretzler M, Anders HJ: Expression and regulation of Toll-like receptors in lupus-like immune complex glomerulonephritis of MRLFas(lpr) mice. Nephrol Dial Transplant 2006; 21:3062-3073.

- 3 Brown HJ, Lock HR, Sacks SH, Robson MG: TLR2 stimulation of intrinsic renal cells in the induction of immune-mediated glomerulonephritis. J Immunol 2006;177:19251931.

\4 Brown HJ, Lock HR, Wolfs TG, Buurman WA, Sacks SH, Robson MG: Toll-like receptor 4 ligation on intrinsic renal cells contributes to the induction of antibody-mediated glomerulonephritis via CXCL1 and CXCL2. J Am Soc Nephrol 2007;18:1732-1739.

-5 Wolfs TG, Buurman WA, van Schadewijk A, de Vries B, Daemen MA, Hiemstra PS, van 't Veer C: In vivo expression of Toll-like receptor 2 and 4 by renal epithelial cells: IFN-gamma and TNF-alpha mediated up-regulation during inflammation. J Immunol 2002;168: 1286-1293.

$>$

-6 Shigeoka AA, Holscher TD, King AJ, Hall FW, Kiosses WB, Tobias PS, Mackman N, McKay DB: TLR2 is constitutively expressed within the kidney and participates in ischemic renal injury through both MyD88-dependent and -independent pathways. J Immunol 2007; 178:6252-6258.

7 Banas MC, Banas B, Hudkins KL, Wietecha TA, Iyoda M, Bock E, Hauser P, Pippin JW, Shankland SJ, Smith KD, Stoelcker B, Liu G, Grone HJ, Kramer BK, Alpers CE: TLR4 links podocytes with the innate immune system to mediate glomerular injury. J Am Soc Nephrol 2008;19:704-713.

> 8 Patole PS, Grone HJ, Segerer S, Ciubar R, Belemezova E, Henger A, Kretzler M, Schlondorff D, Anders HJ: Viral double-stranded RNA aggravates lupus nephritis through Toll-like receptor 3 on glomerular mesangial cells and antigen-presenting cells. J Am Soc Nephrol 2005; 16:1326-1328.

-9 Leemans JC, Stokman G, Claessen N, Rouschop KM, Teske GJ, Kirschning CJ, Akira S, van der Poll T, Weening JJ, Florquin S: Renalassociated TLR2 mediates ischemia/reperfusion injury in the kidney. J Clin Invest 2005;115:2894-2903.

10 Wu H, Chen G, Wyburn KR, Yin J, Bertolino P, Eris JM, Alexander SI, Sharland AF, Chadban SJ: TLR4 activation mediates kidney ischemia/reperfusion injury. J Clin Invest 2007;117:2847-2859.
1 Hang L, Slack JH, Amundson C, Izui S, Theofilopoulos AN, Dixon FJ: Induction of murine autoimmune disease by chronic polyclonal B cell activation. J Exp Med 1983; 157:874-883.

12 Anders HJ, Vielhauer V, Eis V, Linde Y, Kretzler M, Perez de Lema G, Strutz F, Bauer S, Rutz M, Wagner H, Grone HJ, Schlondorff D: Activation of toll-like receptor-9 induces progression of renal disease in MRL-Fas(lpr) mice. FASEB J 2004; 18:534536.

13 Pawar RD, Patole PS, Zecher D, Segerer S, Kretzler M, Schlondorff D, Anders HJ: Tolllike receptor-7 modulates immune complex glomerulonephritis. J Am Soc Nephrol 2006; 17:141-149.

14 Gilkeson GS, Ruiz P, Pippen AM, Alexander AL, Lefkowith JB, Pisetsky DS: Modulation of renal disease in autoimmune NZB/NZW mice by immunization with bacterial DNA. J Exp Med 1996;183:1389-1397.

15 Lafyatis R, Marshak-Rothstein A: Toll-like receptors and innate immune responses in systemic lupus erythematosus. Arthritis Res Ther 2007;9:222.

16 Christensen SR, Shupe J, Nickerson K, Kashgarian M, Flavell RA, Shlomchik MJ: Tolllike receptor 7 and TLR9 dictate autoantibody specificity and have opposing inflammatory and regulatory roles in a murine model of lupus. Immunity 2006;25:417-428. 
-17 Tomosugi NI, Cashman SJ, Hay H, Pusey CD, Evans DJ, Shaw A, Rees AJ: Modulation of antibody-mediated glomerular injury in vivo by bacterial lipopolysaccharide, tumour necrosis factor, and IL-1. J Immunol 1989; 142:3083-3090.

- 18 Pisitkun P, Deane JA, Difilippantonio MJ, Tarasenko T, Satterthwaite AB, Bolland S: Autoreactive B cell responses to RNA-related antigens due to TLR7 gene duplication. Science 2006;312:1669-1672.

$\checkmark 19$ Barrat FJ, Meeker T, Chan JH, Guiducci C, Coffman RL: Treatment of lupus-prone mice with a dual inhibitor of TLR7 and TLR9 leads to reduction of autoantibody production and amelioration of disease symptoms. Eur J Immunol 2007;37:3582-3586.
20 Pawar RD, Ramanjaneyulu A, Kulkarni OP, Lech M, Segerer S, Anders HJ: Inhibition of Toll-like receptor-7 (TLR-7) or TLR-7 plus TLR-9 attenuates glomerulonephritis and lung injury in experimental lupus. J Am Soc Nephrol 2007; 18:1721-1731.

21 Brown HJ, Sacks SH, Robson MG: Toll-like receptor 2 agonists exacerbate accelerated nephrotoxic nephritis. J Am Soc Nephrol 2006;17:1931-1939.

-22 Anders HJ, Banas B, Linde Y, Weller L, Cohen CD, Kretzler M, Martin S, Vielhauer V, Schlondorff D, Grone HJ: Bacterial CpGDNA aggravates immune complex glomerulonephritis: role of TLR9-mediated expression of chemokines and chemokine receptors. J Am Soc Nephrol 2003;14:317-326.
23 Suzuki H, Suzuki Y, Narita I, Aizawa M, Kihara M, Yamanaka T, Kanou T, Tsukaguchi H, Novak J, Horikoshi S, Tomino Y: Toll-like receptor 9 affects severity of IgA nephropathy. J Am Soc Nephrol 2008;19:2384-2395.

-24 Huugen D, Xiao H, van Esch A, Falk RJ, Peutz-Kootstra CJ, Buurman WA, Tervaert JW, Jennette JC, Heeringa P: Aggravation of anti-myeloperoxidase antibody-induced glomerulonephritis by bacterial lipopolysaccharide: role of tumor necrosis factor-alpha. Am J Pathol 2005;167:47-58. 